\title{
Low and heterogeneous prevalence of glucose-6-phosphate dehydrogenase deficiency in different settings in Ethiopia using phenotyping and genotyping approaches
}

Getasew Shitaye $^{1 \dagger}$, Endalamaw Gadisa ${ }^{2 \dagger}$, Lynn Grignard ${ }^{3}$, Girma Shumie ${ }^{2}$, Wakweya Chali²,

Temesgen Menberu ${ }^{2}$, Mulualem Belachew ${ }^{4}$, Getaneh Tegegn ${ }^{5}$, Sagni Challi , Jonathan Curry ${ }^{6}$, Laleta Mahey ${ }^{6}$,

Tsegaye Hailu' ${ }^{2}$, Hassen Mamo ${ }^{7}$, Menakath Menon ${ }^{8}$, Taye Balcha², Abraham Aseffa ${ }^{2}$, Chris Drakeley ${ }^{3}$,

Teun Bousema ${ }^{3,9}$ and Fitsum G. Tadesse $2,9,10^{*}$

\begin{abstract}
Background: 8-Aminoquinolines such as primaquine clear mature Plasmodium falciparum gametocytes that are responsible for transmission from human to mosquitoes and bring radical cure in Plasmodium vivax by clearing dormant liver stages. Deployment of primaquine is thus of relevance for malaria elimination efforts but challenged by the widespread prevalence of glucose-6-phosphate dehydrogenase deficiency (G6PDd) in endemic countries since primaquine in G6PDd individuals may lead to acute haemolysis. In this study, the prevalence of G6PDd was investigated in different settings in Ethiopia using phenotyping and genotyping approaches.
\end{abstract}

Methods: Community and school based cross-sectional surveys were conducted from October to December 2016 in four administrative regions (Gambela, Benishangul Gumuz, Oromia, and Amhara) in Ethiopia. Finger prick blood samples were collected for G6PD enzyme activity using the CareStart ${ }^{\mathrm{TM}}$ G6PD screening test and genotyping of 36 selected single nucleotide polymorphisms (SNPs) located in the G6PD gene and its flanking regions.

Results: Overall, the prevalence of phenotypic G6PDd was 1.4\% (22/1609). For the first time in the Ethiopian population, the African variant (A-) was detected in 3.5\% (7/199) of the limited set of genotyped samples, which were all phenotypically normal. Interestingly, all of these individuals had a variation at the rs2515904 locus. Strong geographical variation was observed for both phenotypic and genotypic G6PDd; three-quarters of the phenotypically G6PDd individuals were detected in Gambela.

Conclusion: A very low prevalence of G6PDd was detected in the present study populations. The presence of the A - variant alongside other G6PD mutants and the patchy distribution of G6PDd indicate that larger studies specifically designed to unravel the distribution of G6PDd at small geographical scale may be needed to tailor malaria elimination efforts in Ethiopia to the local context.

Keywords: Radical cure, P. vivax, G6PD, 8-Aminoquinoline, Haemolysis

\footnotetext{
*Correspondence: fitsum.tadesse@radboudumc.nl; fitsum.girma@aau.

edu.et

${ }^{\dagger}$ Getasew Shitaye and Endalamaw Gadisa contributed equally to this

work

${ }^{2}$ Armauer Hansen Research Institute (AHRI), Addis Ababa, Ethiopia

Full list of author information is available at the end of the article
} 


\section{Background}

The substantial reduction in the global burden of malaria in the last 15 years prompted a renewed interest in malaria elimination [1]. Vector control interventions and case management with effective anti-malarials remain the cornerstones in the fight against malaria [2]. Current antimalarial drugs primarily target the blood stage asexual parasites that are uniquely associated with the signs and symptoms of the disease; with incomplete activity against mature Plasmodium falciparum gametocytes (sexual stages) that are responsible for human-tomosquito transmission [3] and the dormant liver stages (hypnozoites) in Plasmodium vivax that are responsible for multiple relapses weeks or months after the primary infections have resolved [4]. Transmission reduction for both P. vivax and P. falciparum [5] and radical cure in P. vivax [6] are integral parts of the effort to eliminate malaria. Elimination efforts require drugs that not only alleviate symptoms, but also prevent transmission and provide a radical cure [7].

Primaquine $(\mathrm{PQ})$, an 8-aminoquinoline, is the only licensed drug to date that can clear circulating mature gametocytes and the hypnozoites of $P$. vivax $[8,9]$. However, utilization of $\mathrm{PQ}$ is hindered by the association of glucose-6-phosphate dehydrogenase deficiency (G6PDd) and PQ-induced haemolysis [10-12]. The human G6PD gene, located on the X-chromosome [13], codes for a cytosolic enzyme [14] with key roles to produce the reducing equivalent nicotinamide adenine dinucleotide phosphate (NADPH) that is required to protect cells, including red blood cells (RBCs), from oxidizing agents [15]. The G6PD gene has multiple mutant alleles which entail a decrease in enzyme activity; expressing the G6PD deficient (G6PDd) phenotype [16]. In humans, G6PDd is the most common enzyme defect [17] that affects close to 400 million people worldwide [18]. More than 200 different mutations or combinations of mutations that are responsible for gradients of clinical conditions have been identified. The G6PDd variants may remain asymptomatic in the absence of triggers but may result in haemolytic anaemia induced by medications, infection, or ingestion of certain foods due to oxidative stress. Any person with RBC G6PD activity $<30 \%$ of the normal adjusted male median enzyme activity is considered G6PDd while $>80 \%$ is considered normal [19]. Strong regional patterns in the distribution of variants have been reported [20]. The Mediterranean variant (rs5030868) exhibits exceedingly low residual enzyme activity $(<1 \%)$ [21] while the Asian variants are more diverse [22] and could cause adverse effects (5-32\% residual activity) [23]. The common G6PD allelic variants in sub-Saharan Africa are the wild type $G 6 P D^{*} \mathrm{~B}, G 6 P D^{*} \mathrm{~A}+$ (a nondeficient variant, $\mathrm{A} 376 \mathrm{G}$ ), and $G 6 P D^{*} \mathrm{~A}-$ (the deficient variant, A376G plus G202A) [16, 17]. G6PD*A- variants with substitutions at G542T, G680T or T968C were also identified [24, 25]. Compared to the Mediterranean (rs5030868) and South East Asian variants, the African variant is generally considered to result in a milder degree of deficiency and less severe haemolysis [20]; although incidents of life-threatening haemolysis have been reported for G6PD A- [26].

Recent estimates indicated that the number of new cases and deaths attributable to malaria in Ethiopia declined by $96.5 \%$ and $88.7 \%$, respectively, between 1990 and 2015 [27]. Unlike most of Africa, P. vivax is uniquely highly prevalent in the country. Ethiopia contributed towards $10 \%$ of the global vivax malaria cases in 2016 [28]. Ethiopia set an ambitious plan of eliminating malaria from selected low-endemic districts by 2020 [29]. The World Health Organization (WHO) recommends single low dose PQ as a $P$. falciparum gametocytocide and a 14-days course for radical cure of $P$. vivax [30]. PQ, which was used in the country for over a quarter of a century before it was removed from treatment regimens for no documented reasons in 1990 [31], is currently reincorporated into the treatment policy of Ethiopia in elimination settings. However, very little evidence is available on the distribution of G6PDd in Ethiopia and the associated mutations. With the move to deploy PQ widely it is timely and important to generate evidence on G6PDd in the Ethiopian population. In this study, G6PDd was investigated in different malaria endemic settings in the Ethiopian population using enzymatic assay and multiple allelic forms of G6PD were analysed by genotyping.

\section{Methods}

\section{Study sites and population}

Community and school based cross-sectional surveys were conducted from October to December 2016 to investigate G6PDd by phenotyping and genotyping approaches in Ethiopia. Nine Woredas (administrative unit equivalent to district) representing different malaria endemic settings were selected from four Regional States (Gambela, Benishangul Gumuz, Oromia and Amhara Regions). Community based cross-sectional surveys were conducted in Abobo and Lare Woredas of Gambela, Meng and Mao-komo Woredas of Benishangul Gumuz, Gomma and Adama Woredas of Oromia Regional States, Fig. 1. Community mobilization was conducted prior to the study. Residents who were willing to participate in the study were requested to come to the study clinic the next day to donate finger prick blood samples. School based surveys were conducted in Amhara Regional State in Jawi (Jawi general primary school), North Achefer (Ahuri primary school), and Bahir Dar Zuria (Andassa primary 


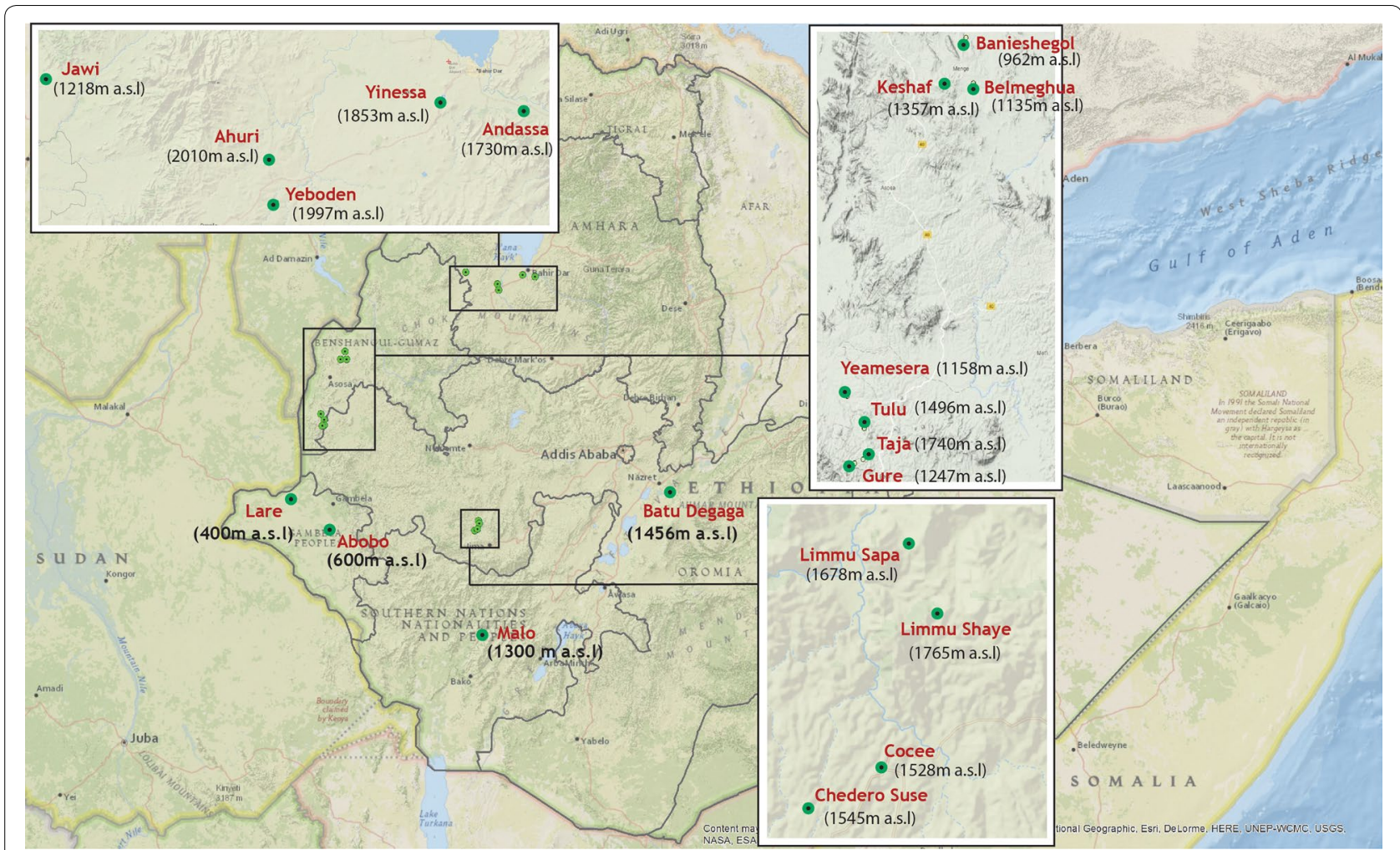

Fig. 1 Map of study sites. Community surveys were conducted in Lare and Abobo Woredas of Gambela Regional State, Meng (in Banieshegol, Keshaf and Belmeguha Kebeles) and Maokomo (in Yeamesera, Tulu, Taja and Gure Kebeles) Woredas of Benishangul-Gumuz Regional State, Goma (in Limmu Sapa, Limmu Shaye, Cocee and Chedero Suse Kebeles) and Adama (Batu Degaga Kebele) Woredas of Oromia Regional State. School surveys were conducted in South Achefer (Yinessa and Yeboden Kebeles), Bahir Dar Zuria (Andassa Kebele), and Jawi Woredas in Amhara Regional State. Elevation of each site (meters above sea level, m.a.s.l) is indicated in brackets

school) Woredas, Fig. 1. School based surveys were conducted to estimate parasite prevalence [32] and performed as routinely done using methods developed by Brooker and colleagues to obtain an age-balanced set of samples [33].

\section{Blood sample collection and G6PD phenotypic screening}

Socio-demographic data were recorded using a pretested questionnaire and individuals were requested to donate a finger prick blood sample $(\sim 300 \mu \mathrm{l})$ that was used for the assessment of activity of the G6PD enzyme using the CareStart ${ }^{\mathrm{TM}}$ G6PDd screening test (Access Bio, Inc., New Jersey, USA). The remaining blood was used to prepare dried blood spots (DBS) on WhatMann 3MM (Whatman, Maidstone, UK) filter papers [32]. DBS samples were air dried, individually packed in a cardboard that had the study code and stored in zip-locked plastic bags containing self-indicating silica gel desiccant beads (Geejay Chemicals Ltd) and transported to the laboratory at AHRI and stored at $-20{ }^{\circ} \mathrm{C}$ freezer until processed.

\section{DNA extraction and single nucleotide polymorphism (SNP) genotyping}

Genomic DNA was extracted using saponin-chelex dual extraction procedure as described previously [34] from a $6 \mathrm{~mm}$ diameter punch that was eluted in $150 \mu \mathrm{l}$ of a $6 \%$ Chelex in DNase/RNase free water solution and stored at $-20{ }^{\circ} \mathrm{C}$ until further use. Thirty-six SNPs located in the $G 6 P D$ gene and its flanking regions were selected from those identified in the literature and on public databases based on criteria described earlier [35-37]. Samples that were found deficient with the enzymatic assay (22) and a subset of 211 G6PD phenotypically normal samples, randomly selected from all study sites, were genotyped using Kompetitive Allele Specific PCR (KASP ${ }^{\mathrm{TM}}$ ) (LGC Genomics, UK). For the 36 putative SNP sequences identified, KASP assays were designed, comprising two allele-specific forward primers, and one common reverse primer (Additional file 1). Primer designs were using sequence derived from NCBI dbSNP and each sequence screened for potential surrounding polymorphism using population information held [37]. Alternative primers were designed if another surrounding polymorphism had an 


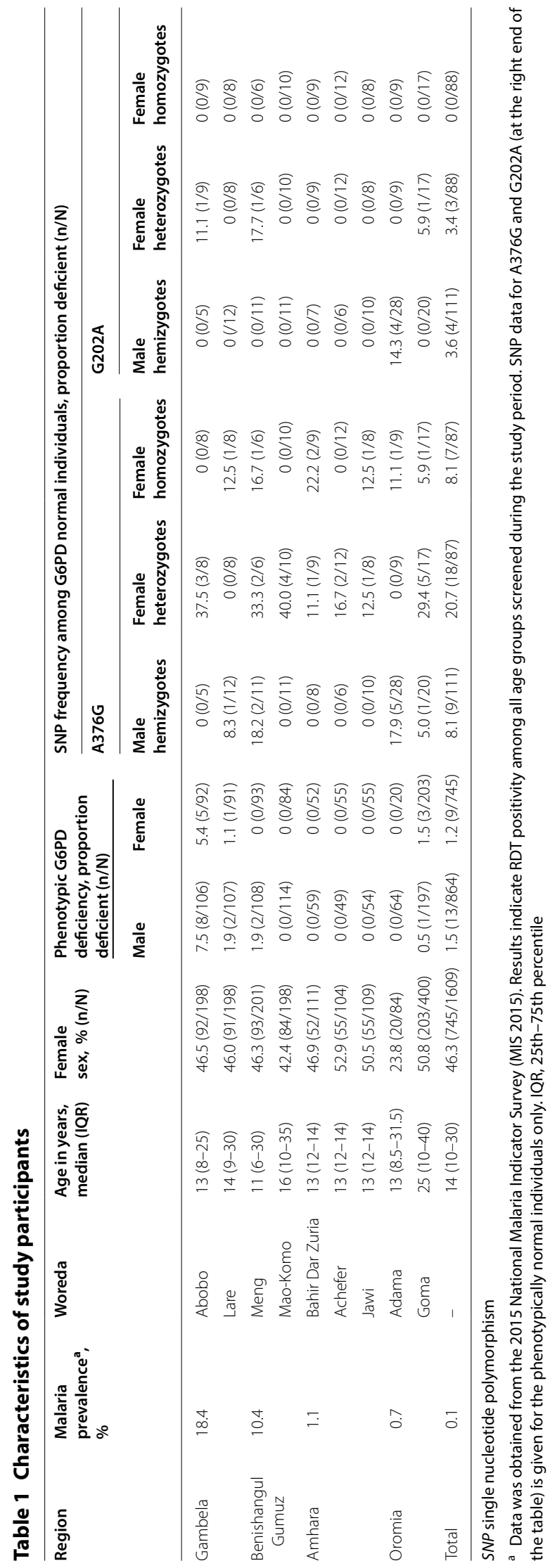


alternative allele frequency greater than 1\% (Additional file 2). If was not possible to avoid, then ambiguities were incorporated into the primer in order to minimize any potential impact on assay performance. Genotyping reactions were performed in duplicate using the IntelliQube (LGC, Douglas Scientific, USA) to complete all sample DNA arraying, reagent dispensing, plate sealing and fluorescence detection steps for SNP genotyping as described in detail before [37].

\section{Data analysis}

Questionnaire data was checked for completeness and double-entered along with all laboratory results in RedCap (Vanderbilt University, USA) [38]. Statistical analysis was conducted using STATA 13 (StataCorp, TX, USA) and Graph Pad Prism 5.0 (Graph Pad Software Inc., CA, USA). Computation of Linkage Disequilibrium (LD) and haplotype analysis was done using Haploview version 4.2 Software as stated elsewhere [39]. As a quality control, SNPs with greater than $10 \%$ missing genotype calls and more than $2 \%$ of male genotype calls that were (falsely) called heterozygous were excluded from analysis including individuals with greater than $20 \%$ of the SNP data missing (Additional files 1 and 3). Monomorphic SNPs and those with less than $1 \%$ frequency were also excluded from LD and haplotype analysis. No sample size calculations were performed prior to data collection; $95 \%$ confidence intervals $(\mathrm{CI})$ were presented for all prevalence estimates.

\section{Results}

A total of 1609 individuals with a median age of 14 years (interquartile range [IQR]: 10-30); of whom 46.3\% (745/1609) were females, participated in this study. The overall phenotypic G6PDd detected by the CareStart ${ }^{\mathrm{TM}}$ G6PDd screening test was 1.4\% (95\% confidence interval [CI] 1.0-2.1), with considerable variation between study sites. Of the 22 G6PDd individuals 16 were from Gambela Regional State: 6.6\% (95\% CI 3.5-11.0) of participants from Abobo and 1.5\% (95\% CI 0.3-4.4) of participants from Lare Woredas (Table 1). Of the phenotypically deficient individuals 9 were females.

A total of 14 SNPs that did not meet the quality control were excluded from further analysis (Additional files 1 and 3): 1 SNP with $>10 \%$ missing genotype calls (rs35228794) and 13 SNPs with more than 2\% of male genotype calls that were (falsely) called heterozygous (rs766420, rs915941, rs915942, rs1894260, rs2515906, rs1050757, rs2230037, b36_153426256, rs4898389, rs5986877, rs7879049, rs7053878, rs2004651). Additionally, 28 individuals with more than $20 \%$ missing calls were also excluded. Out of the 22 high quality G6PD SNPs, 6 were found monomorphic [including the well-known variants in Africa rs76723693 (968T $>$ C); rs5030872 $(542 \mathrm{~A}>\mathrm{T})$; rs137852328 (680) and the Mediterranean variant (rs5030868)]. One SNP (rs12389569) had $<1 \%$ minor allele frequency (MAF) (Table 2). Thus, further analyses focused on 15 SNPs that passed the quality control. Four of these SNPs are located in the G6PD gene (2 synonymous and 2 missense variants) and the other in flanking regions of the gene; i.e. 3 immediately downstream of G6PD gene and the remaining were intergenic (1), intron (5), non-coding exon (2) and 5' UTR variants (1).

Among the common SNPs genotyped, the A376G mutation was detected in 34 individuals $(17.1 \%, 95 \% \mathrm{CI}$ 12.1-23.0) (Tables 1 and 2); 9 (8.1\%, 95\% CI 3.8-14.8) hemizygous males, 7 (8.0\%, 95\% CI 3.3-15.9) homozygous females, and 18 (20.7\%, 95\% CI 12.7-30.7) heterozygous females. In three male participants, a heterozygous signal was observed and confirmed upon repeating the assay. The G202A mutation was detected in 7 (3.5\%, 95\% CI 1.4-7.1) individuals of whom 4 (3.6\%, 95\% CI 1.0-9.0) were hemizygous males and the remaining $(3.4 \%, 95 \% \mathrm{CI}$ 0.7-9.6) were heterozygous females. All G202A hemizygous males were hemizygous for the A376G mutation and of the G202A heterozygous females 2 were homozygous and 1 was heterozygous for the A376G mutation. The African variant was not detected among the phenotypically deficient individuals, only one male phenotypically deficient individual had hemizygous A376G variant. That same individual was hemizygous for rs 28470352 , rs5986990, rs762515, rs111827785, and rs60030796 variants. The remaining phenotypically deficient individuals had wild type variants for all the SNPs analyzed in this study. Taken together, all individuals that carried the G202A variant also carried the A376G mutations and the A- African variant could not explain the phenotypic variation observed in the present study. All individuals with the A- variant (male hemizygotes and female heterozygotes) were found carrying a variation at the rs 2515904 locus (4.6\%, 95\% CI 2.1-8.5). Like the African variant, the variation at the rs2515904 locus was detected only in phenotypically normal individuals, Table 2 . In a similar fashion to the geographic distribution of the phenotypic G6PDd, 4/7 (57.1\%) of the A- variants were detected from Adama; they were detected from two families each and two of them were brothers (Table 1). The G202A variant showed high geographic clustering, whereas the A376G mutation was uniformly distributed across all study sites.

Linkage disequilibrium (LD) plot could be constructed only for the whole population. It was not stratified based on gender, ethnicity and study setting due to small samples genotyped. One LD block was constructed that consisted of rs28470352, rs73573478, rs5986990, rs1050829 
Table 2 Single nucleotide polymorphisms detected in the study population

\begin{tabular}{|c|c|c|c|c|c|c|c|c|}
\hline \multirow[t]{2}{*}{$\begin{array}{l}\text { Reference SNP } \\
\text { identifier }\end{array}$} & \multirow[t]{2}{*}{$\begin{array}{l}\text { Chromosome } \\
\text { position }\end{array}$} & \multicolumn{3}{|c|}{$\begin{array}{l}\text { SNP frequency by sex, proportion deficient } \\
(\mathrm{n} / \mathrm{N})\end{array}$} & \multicolumn{2}{|c|}{$\begin{array}{l}\text { Total SNP frequency, } \\
\text { proportion deficient }(n / N)\end{array}$} & \multirow{2}{*}{$\begin{array}{l}\text { Ancestral: } \\
\text { alternate } \\
\text { allele }\end{array}$} & \multirow[t]{2}{*}{ Function } \\
\hline & & $\begin{array}{l}\text { Male } \\
\text { hemizygotes }\end{array}$ & $\begin{array}{l}\text { Female } \\
\text { homozygotes }\end{array}$ & $\begin{array}{l}\text { Female } \\
\text { heterozygotes }\end{array}$ & $\begin{array}{l}\text { Hemizygous/ } \\
\text { homozygous }\end{array}$ & Heterozygous & & \\
\hline rs28470352 & $153,753,490$ & $8.3(10 / 121)$ & $5.8(5 / 87)$ & $20.7(18 / 87)$ & $5.8(12 / 208)$ & $10.1(21 / 208)$ & $\mathrm{T}: \mathrm{A}$ & $\begin{array}{l}\text { Intergenic } \\
\text { variant }\end{array}$ \\
\hline rs61042368 & $153,755,336$ & $5.0(6 / 120)$ & $1.2(1 / 86)$ & $1.2(1 / 86)$ & $2.4(5 / 206)$ & $1.5(3 / 206)$ & $\mathrm{G}: \mathrm{A}$ & $\begin{array}{l}\text { Downstream } \\
\text { gene vari- } \\
\text { ant }\end{array}$ \\
\hline rs12389569 & $153,757,734$ & $0(0 / 120)$ & $0(0 / 85)^{*}$ & $2.4(2 / 85)$ & $0(0 / 205)$ & $1.0(2 / 205)$ & $\mathrm{G}: \mathrm{A}$ & $\begin{array}{l}\text { Downstream } \\
\text { gene vari- } \\
\text { ant }\end{array}$ \\
\hline b36_153413623 & $153,760,429$ & $1.7(2 / 121)$ & $2.4(2 / 85)$ & $3.5(3 / 85)$ & $1.9(4 / 206)$ & $1.5(3 / 206)$ & G:A & $\begin{array}{l}\text { Synonymous } \\
\text { variant }\end{array}$ \\
\hline rs2230036 & $153,760,953$ & $5.9(7 / 119)$ & $1.2(1 / 86)$ & $1.2(1 / 86)$ & $2.4(5 / 205)$ & $2.0(4 / 205)$ & $C: T$ & $\begin{array}{l}\text { Synonymous } \\
\text { variant }\end{array}$ \\
\hline rs73573478 & $153,761,564$ & $8.9(11 / 123)$ & $2.2(2 / 90)$ & $4.4(4 / 90)$ & $4.7(10 / 213)$ & $3.3(7 / 213)$ & G:A & $\begin{array}{l}\text { Non coding } \\
\text { exon vari- } \\
\text { ant }\end{array}$ \\
\hline rs5986990 & $153,761,628$ & $7.8(9 / 115)$ & $5.9(5 / 85)$ & $21.2(18 / 85)$ & $6.0(12 / 200)$ & $10.0(20 / 200)$ & $\mathrm{G}: \mathrm{A}$ & $\begin{array}{l}\text { Non coding } \\
\text { exon vari- } \\
\text { ant }\end{array}$ \\
\hline rs2515905 & $153,762,075$ & $3.3(4 / 120)$ & $1.2(1 / 87)$ & $4.6(4 / 87)$ & $2.4(5 / 207)$ & $1.9(4 / 207)$ & $\mathrm{G}: \mathrm{A}$ & Intron variant \\
\hline rs2515904 & $153,762,771$ & $3.3(4 / 121)$ & $1.2(1 / 87)$ & $4.6(4 / 87)$ & $2.4(5 / 208)$ & $1.9(4 / 208)$ & $G: C$ & $\begin{array}{l}\text { Intron vari- } \\
\text { ant }\end{array}$ \\
\hline rs1050829 & $153,763,492$ & $8.3(10 / 121)$ & $7.9(7 / 89)$ & $20.2(18 / 89)$ & $6.7(14 / 210)$ & $10.0(21 / 210)$ & $A: G$ & $\begin{array}{l}\text { Missense } \\
\text { variant }\end{array}$ \\
\hline rs1050828 & $153,764,217$ & $3.3(4 / 120)$ & $0(0 / 90)$ & $3.3(3 / 90)$ & $1.9(4 / 210)$ & $1.4(3 / 210)$ & $G: A$ & $\begin{array}{l}\text { Missense } \\
\text { variant }\end{array}$ \\
\hline rs762515 & $153,764,528$ & $7.7(9 / 117)$ & $5.8(5 / 86)$ & $20.0(17 / 86)$ & $5.9(12 / 203)$ & $9.4(19 / 203)$ & $\mathrm{T}: \mathrm{C}$ & Intron variant \\
\hline rs762516 & $153,764,663$ & $3.3(4 / 121)$ & $1.2(1 / 87)$ & $4.6(4 / 87)$ & $2.4(5 / 208)$ & $1.9(4 / 208)$ & C:T & Intron variant \\
\hline rs113492957 & $153,773,062$ & $9.2(11 / 120)$ & $2.3(2 / 86)$ & $3.5(3 / 86)$ & $4.9(10 / 206)$ & $2.9(6 / 206)$ & $C: T$ & Intron variant \\
\hline rs111827785 & $153,775,785$ & $18.2(22 / 121)$ & $9.6(8 / 83)$ & $24.1(20 / 83)$ & $12.8(26 / 204)$ & $11.8(24 / 204)$ & $\mathrm{T}: \mathrm{C}$ & $5^{\prime} \cup T R$ \\
\hline rs60030796 & $153,836,171$ & $5.0(6 / 119)$ & $4.6(4 / 87)$ & $12.6(11 / 87)$ & $3.4(7 / 206)$ & $6.8(14 / 206)$ & $A: G$ & $\begin{array}{l}\text { Downstream } \\
\text { gene vari- } \\
\text { ant }\end{array}$ \\
\hline
\end{tabular}

SNP single nucleotide polymorphism

The following SNPs were monomorphic and excluded from further analysis: rs5030869, rs76723693 (968), rs137852328 (680), rs5030868, rs5030872 (542), and rs137852318. *SNP with minor allele frequency < 1\% and was omitted from further analysis. Italicized are the well-known rs1050828 (G202A) and rs1050828 (A376G) African polymorphisms. Bolded and italicized ( $r$ 2515904) indicates a SNP that was detected in individuals who carried the 376 and 202 variants

(376), rs762515, rs113492957, rs111827785, and rs60030796 (Fig. 2). Pairwise LD was high in this study (combined gender result was median 1.00; range 0.641.00). Generated haplotypes were listed in Table 3.

\section{Discussion}

A very low frequency $(1.4 \%, 22 / 1609)$ of phenotypically evident G6PDd was detected in this study. For the first time in published literature, the African variant $(\mathrm{A}-)$ was detected in an Ethiopian population. Of the genotyped samples 3.5\% (7/199) were A- variants (4 hemizygous males and 3 homozygous females) and consisted of both the G202A and the A376G mutations. Three quarters of the phenotypically deficient individuals were from one Region, Gambela. None of the phenotypically deficient individuals were found to have the A - variant. Uniquely, all individuals with the A- variant were found carrying a variation at the rs2515904 locus that was previously found in perfect LD with the A- variant and was reported protective against clinical malaria [40-42].

The high prevalence of G6PDd detected by the phenotypic assay in Gambela Region is in line with a recent finding of $7.3 \%$ phenotypic deficiency in the same region [43] and with an earlier finding (of 4.0\%) among tribes in Eritrea [44]. According to the 2015 National Malaria Indicator Survey, Gambela Region reported the highest 


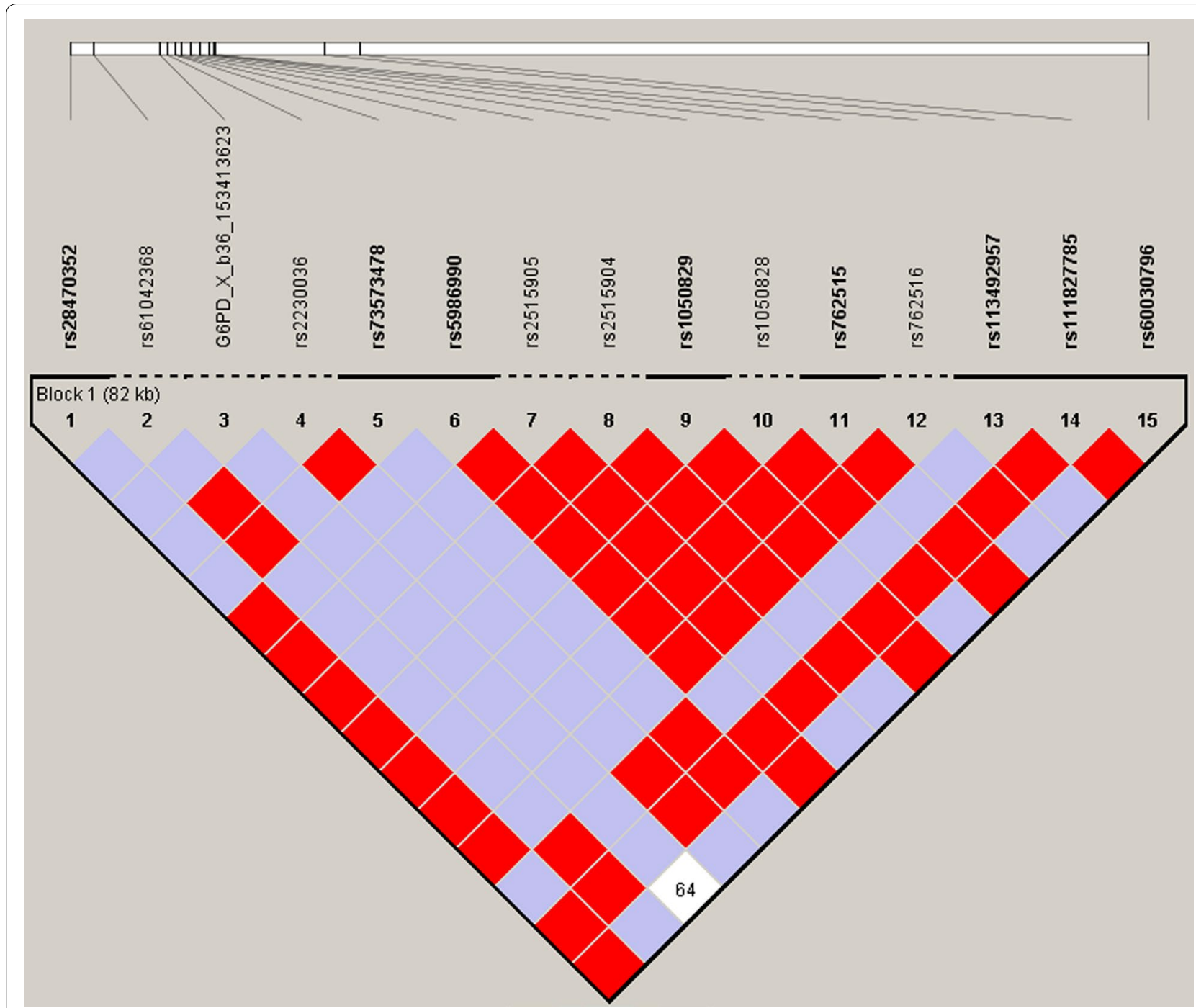

Fig. 2 Linkage disequilibrium (LD) plot estimated by Haploview. The numbers in the diamonds are $D^{\prime}$ values, with red and no numbers representing a $D^{\prime}$ value of 1 for the corresponding pair of variants. Strong LD is indicated by dark gray, whereas light gray and white indicate uninformative and low confidence values, respectively. LD blocks were created with the default algorithm in Haploview that creates $95 \%$ confidence bounds on $D^{\prime}$ considered to be in strong LD, where $95 \%$ of the comparisons made are informative

Table 3 Haplotype frequencies in phenotypically normal participants

\begin{tabular}{ll}
\hline Haplotypes & $\begin{array}{l}\text { Haplotype } \\
\text { frequencies }\end{array}$ \\
\hline TGGATCTA & 0.812 \\
AGAGCCCG & 0.076 \\
TAGATTCA & 0.056 \\
AGAGCCCA & 0.045 \\
TGGATCCA & 0.011 \\
\hline
\end{tabular}

SNPs refer to the following corresponding to Table 2: rs28470352, rs73573478, rs5986990, rs1050829 (376), rs762515, rs113492957, rs111827785, rs60030796. 376 are bolded prevalence of malaria in Ethiopia $(18.4 \%$ by RDT compared to $0.1 \%$ country wide prevalence) [45] and it is one of the areas in the country with historically stable malaria transmission [46-48]. This might be attributable to its location within the humid tropical region of the country ( $<500$ masl), settlement and land-use changes [46], and periodic flooding [49]. The geographic overlap of the high malaria and G6PDd observed in this study is supportive of the long-standing claim that this mutation was naturally selected by malaria [50].

Phenotypically evident deficiencies detected in G6PDd individuals often have matched genetic background (often of point mutation or haplotypes). A to G 
substitution mutation at the rs1050829 locus (also known as A376G, exon 5) is associated with reduced production of G6PD enzyme that causes a milder variant, G6PD A+, resulting in $80 \%$ residual enzyme activity compared to normal values [51]. The G6PD deficient genotype A- is typically defined by the possession of both the rs 1050829 (A376G) allele and G to A substitution at the rs1050828 locus (also known as G202A, exon 4). Type A- mutations cause on average $12 \%$ residual G6PD activity [51]. Type A- is predominantly found in people who have African ancestry (and referred to as the African variant). In line with the hypothesis that the mutation leading to the African variant (G202A) might have arisen on a chromosome that carried the silent mutation (A376G), all G202A mutations in the present study were detected in individuals carrying the A376G mutation. Most of the phenotypically G6PD deficient individuals in the present study had no SNPs detected in the G6PD gene. There was one phenotypically deficient individual who had the A376G, together with variations at rs28470352, rs5986990, rs762515, rs111827785, and rs60030796 loci, but not the G202A. All other SNPs tested in this study were monomorphic for phenotypically deficient individuals, which might be explained by G6PD mutations in the Ethiopian population that were not tested in the present study $[52,53]$. A relevant shortcoming of the current study was that selected populations of G6PDd and G6PD normal individuals were included in the genotyping assays; the unavailability of an unbiased population makes it challenging to derive meaningful allele frequencies. An additional major limitation was that the sample size was too small to estimate the frequencies of phenotypic G6PDd and mutations in the G6PD gene, as well as their associations with RDT performance with precision. The very small sample size, especially with the genotyping analysis, could also have contributed to the observed geographic clustering. The detection of the African variant of G6PDd in the present study and the predominant geographic pattern of patchy distribution of G6PDd underline the need for larger studies to have complete picture of the nationwide distribution of the deficiency to help build a resilient programmatic approach in the move towards eliminating malaria in Ethiopia.

With the limited number of genotyped samples from different study sites, 3.5\% (7/199) of genotyped samples were of the A- variant. This contrasts with, previous studies conducted in different settings in Southwest Ethiopia, South Omo [34] and Jimma [52] that did not detect the African $\mathrm{A}-$ variant among their Ethiopian populations. One of the unique differences may lie in the study population; the previous studies were all done on clinical patients [52]; possibly missing variants that infer protection from clinical malaria. The other possible explanation is that these studies only included single settings, possibly missing the strong regional patterns in the distribution of G6PD variants [20] and differences in ethnic background $[43,54,55]$ as demonstrated in the present study. Apart from this the results from this study on the A376G variants $(17.1 \%, 34 / 199)$ are in line with a recent finding from Jimma (23.3\%) [52]. The Mediterranean variant (rs5030868, C563T) was not detected in any of these studies, including the present study $[34,52]$. Allelic heterogeneity, the phenomenon in which different mutations at the same locus cause the same phenotype, might have contributed to the discrepancy reported in previous studies as most of them relied on restriction digestion [34]. Allelic variations can arise as a result of natural selection processes, exogenous mutagens, genetic drift, or genetic migration.

Mutations of G6PD can cause varying degrees of G6PDd and thus severity of haemolysis may vary. The patterns of oxidative haemolysis are well characterized in hemizygous males and homozygous females; whereas, heterozygous females may exhibit symptoms to varying degrees due to random inactivation of $\mathrm{X}$-chromosomes [56]. The expression of G6PDd differs markedly among female heterozygotes as their RBC populations are variable mosaics of deficient and normal cells [57] and can potentially be misclassified by currently available phenotypic diagnostic approaches. Although rapid tests that can be used at point-of-care were recently introduced for the diagnosis of G6PDd $[58,59]$, they may fail to accurately identify individuals with intermediate deficiency (30-80\% normal enzymatic activity, mainly heterozygote females) [19]. Heterozygous females who test normal or not deficient in qualitative G6PD screening tests can still experience substantial hemolysis [56]. A quantitative technique is thus required to detect the intermediate levels of G6PD activity but this is not yet available as a point-of-care test and will have cost and logistics implications to overcome when developed and scaled-up [60, 61].

A single low dose PQ as a gametocytocide in P. falciparum malaria and a 14-day course for radical cure in $P$. vivax is recommended by the $\mathrm{WHO}$ and this is being incorporated into country treatment policies. Most $P$. vivax malaria-endemic countries recommend $\mathrm{PQ}$ treatment without prior G6PD testing [62]. The WHO malaria treatment guidelines address the 14-day course for preventing relapse with caution, including a statement that good practice requires that the G6PD status of patients should be ascertained prior to administration of PQ [19]. On top of costs associated with deployment of G6PDd diagnostic tests, the largest challenge is the lack of standardized point-of-care diagnostic tests to-date to mitigate the risk of haemolysis associated with drugs like PQ. 
Alternative strategies need to be explored in the Ethiopian settings where the frequency of G6PDd is very low and varies substantially between sites; blanket deployment of diagnostic tests might not be cost-effective. Strategies such as directly observed treatment (DOT), close medical supervision for potential PQ-induced haemolysis, as in tuberculosis care [63], using its extensive health extension workers program (with counseling on how to recognize symptoms and signs of haemolytic anaemia) or approaches tailored to local context considering the varying geographic distribution of the deficiency could be plausible options.

\section{Conclusion}

Prevalence of G6PDd is generally very low and varies substantially between settings in Ethiopia. The country could potentially benefit from tailored deployment of 8 -aminoquinolines like PQ. This study supports the evidence that the incorporation of PQ into national treatment guidelines for $P$. falciparum malaria would likely pose little risk to the population due to the very low genotypic and phenotypic prevalence of G6PDd. Despite the low prevalence, alternative strategies including DOT or at least better screening for G6PDd, particularly among females, who are likely to be phenotypically normal by Carestart G6PD screening test, but may experience substantial haemolysis if they were genotypically female heterozygous, need to be considered to prevent $P$. vivax relapse.

\section{Additional files}

Additional file 1. Flow chart for genotyping and data analysis. Indicated in a) is the schematic presentation of the quality check for data analysis and in $b$ ) is the genotyping procedure.

Additional file 2. KASP Primer sequences.

Additional file 3. Genotyping overview.

\section{Authors' contributions}

EG, TB, CD, and FGT conceived the project. GeS, EG, GiS, WC, TM, MB, GT, $\mathrm{SC}, \mathrm{TH}, \mathrm{HM}, \mathrm{MM}, \mathrm{TB}, \mathrm{AA}$, and FGT planned and executed the field work and participated in discussions during the study. GeS, EG, LG, JC, LM, and FGT did the lab works. FGT analysed results and drafted the manuscript. All authors critically commented on the draft manuscript. All authors read and approved the final manuscript.

\section{Author details}

1 Department of Biomedical Sciences, School of Medical Sciences, Bahir Dar University, Bahir Dar, Ethiopia. ${ }^{2}$ Armauer Hansen Research Institute (AHRI), Addis Ababa, Ethiopia. ${ }^{3}$ Department of Immunology and Infection, London School of Hygiene and Tropical Medicine, London, UK. ${ }^{4}$ Department of Biomedical Sciences, School of Medical Sciences, Wolkite University, Wolkite, Ethiopia. ${ }^{5}$ Department of Biomedical Sciences, Semera University, Semera, Ethiopia. ${ }^{6}$ Genomics Division, LGC Group Itd, Hertfordshire, UK. ${ }^{7}$ Department of Microbial, Cellular and Molecular Biology, College of Natural Sciences, Addis Ababa University, Addis Ababa, Ethiopia. ${ }^{8}$ Department of Biochemistry, School of Medical Sciences, Addis Ababa University, Addis Ababa, Ethiopia. ${ }^{9}$ Radboud Institute for Health Sciences, Radboud University Medical Centre, Nijmegen,
The Netherlands. ${ }^{10}$ Institute of Biotechnology, Addis Ababa University, PO Box 109, Addis Ababa, Ethiopia.

\section{Acknowledgements}

We would like to thank all study participants for their willingness to take part in this study. We thank the school principals, teachers, regional and district health bureau officials, health extension workers, lab technicians, nurses, and community leaders for their assistance during sample collection. We thank Elias F. Mekuriaw for constructing the map.

\section{Competing interests}

The authors declare that they have no competing interests.

\section{Availability of data and materials}

Data supporting findings of the study is made available at the repositories of the Armauer Hansen Research Institute, Addis Ababa, Ethiopia and the Radboud University Medical Center, Nijmege, The Netherlands.

\section{Consent for publication}

Consent for publication of data generated from the study was obtained from all study participants.

\section{Ethics approval and consent to participate}

This study was part of a project that had previously been reported before [64]. The present study was reviewed and approved by the ethics review committee at the Department of Biochemistry, School of Medical Sciences, Addis Ababa University (Ref.No.SOM/DRERC/BCH005/2009). Informed written consent was obtained from each individual and/or parents/guardians, in case of minors.

\section{Funding}

This work was supported by grants from the Netherlands Organization for Scientific Research (Vidi fellowship) [NWO Project Number 016.158.306] and fellowship from the European Research Council [ERC-2014-StG 639776] to TB; the Bill \& Melinda Gates foundation [INDIE; OP P1173572] to TB and CD; the Netherlands organization for international cooperation in higher education (Nuffic) [Grant Number NFP-PhD.14/150] to FGT and the Armauer Hansen Research Institute (via its core funding from Norwegian Agency for Development Cooperation and Swedish International Development Cooperation).

\section{Publisher's Note}

Springer Nature remains neutral with regard to jurisdictional claims in published maps and institutional affiliations.

Received: 22 June 2018 Accepted: 30 July 2018

Published online: 02 August 2018

\section{References}

1. WHO. World malaria report 2017. World Health Organization, Geneva. 2017. http://www.who.int/malaria/publications/world-malaria-repor t-2017/en/. Accessed 25 Feb 2018

2. Huijben S, Paaijmans KP. Putting evolution in elimination: winning our ongoing battle with evolving malaria mosquitoes and parasites. Evol Appl. 2018;11:415-30.

3. Bousema T, Drakeley C. Epidemiology and infectivity of Plasmodium falciparum and Plasmodium vivax gametocytes in relation to malaria control and elimination. Clin Microbiol Rev. 2011;24:377-410.

4. Baird JK, Schwartz E, Hoffman SL. Prevention and treatment of vivax malaria. Curr Infect Dis Rep. 2007:9:39-46.

5. Alonso PL, Brown G, Arevalo-Herrera M, Binka F, Chitnis C, Collins F, et al. A research agenda to underpin malaria eradication. PLoS Med. 2011:8:e1000406

6. Cox FE. History of the discovery of the malaria parasites and their vectors. Parasit Vectors. 2010;3:5

7. Flannery EL, Chatterjee AK, Winzeler EA. Antimalarial drug discoveryapproaches and progress towards new medicines. Nat Rev Microbiol. 2013;11:849. 
8. Graves PM, Gelband H, Garner P. Primaquine or other 8-aminoquinoline for reducing Plasmodium falciparum transmission. Cochrane Database Syst Rev. 2015. https://doi.org/10.1002/14651858.CD008152.pub4.

9. Betuela I, Robinson LJ, Hetzel MW, Laman M, Siba PM, Bassat Q, et al. Primaquine treatment for Plasmodium vivax-an essential tool for malaria control and elimination in Papua New Guinea. P N G Med J. 2014;57:68.

10. Ashley EA, Recht J, White NJ. Primaquine: the risks and the benefits. Malar J. 2014;13:418

11. Gonçalves BP, Tiono AB, Ouédraogo A, Guelbéogo WM, Bradley J, Nebie l, et al. Single low dose primaquine to reduce gametocyte carriage and Plasmodium falciparum transmission after artemether-lumefantrine in children with asymptomatic infection: a randomised, double-blind, placebo-controlled trial. BMC Med. 2016;14:40.

12. Domingo GJ, Satyagraha AW, Anvikar A, Baird K, Bancone G, Bansil P, et al. G6PD testing in support of treatment and elimination of malaria: recommendations for evaluation of G6PD tests. Malar J. 2013;12:391.

13. Minucci A, Giardina B, Zuppi C, Capoluongo E. Glucose-6-phosphate dehydrogenase laboratory assay: how, when, and why? IUBMB Life. 2009;61:27-34.

14. Kumar P, Yadav U, Rai V. Prevalence of glucose-6-phosphate dehydrogenase deficiency in India: an updated meta-analysis. EJMHG. 2016;17:295-302

15. Stanton RC. Glucose-6-phosphate dehydrogenase, NADPH, and cell survival. IUBMB Life. 2012;64:362-9.

16. Shah SS, Macharia A, Makale J, Uyoga S, Kivinen K, Craik R, et al. Genetic determinants of glucose-6-phosphate dehydrogenase activity in Kenya. BMC Med Genet. 2014;15:93.

17. Ouattara AK, Bisseye C, Bazie BVJTE, Diarra B, Compaore TR, Djigma F, et al. Glucose-6-phosphate dehydrogenase (G6PD) deficiency is associated with asymptomatic malaria in a rural community in Burkina Faso. Asian Pacific J Trop Biomed. 2014:4:655-8.

18. Luzzatto L, Nannelli C, Notaro R. Glucose-6-phosphate dehydrogenase deficiency. Hematol Oncol Clin. 2016;30:373-93.

19. WHO. Guidelines for the treatment of malaria. World Health Organization, Geneva. 2015. http://apps.who.int/iris/bitstream/handle/10665/16244 1/9789241549127_eng.pdf?sequence=1. Accessed 05 May 2018.

20. Howes RE, Piel FB, Patil AP, Nyangiri OA, Gething PW, Dewi M, et al. G6PD deficiency prevalence and estimates of affected populations in malaria endemic countries: a geostatistical model-based map. PLoS Med. 2012;9:e1001339.

21. Piomelli S, Corash LM, Davenport DD, Miraglia J, Amorosi EL. In vivo lability of glucose-6-phosphate dehydrogenase in Gd A- and Gd Mediterranean deficiency. J Clin Invest. 1968;47:940-8.

22. Tang T, Huang C, Huang M, Tam K, Yeh C, Tang C. Diverse point mutations result in glucose-6-phosphate dehydrogenase (G6PD) polymorphism in Taiwan. Blood. 1992;79:2135-40.

23. Louicharoen C, Patin E, Paul R, Nuchprayoon I, Witoonpanich B, Peerapittayamongkol C, et al. Positively selected G6PD-Mahidol mutation reduces Plasmodium vivax density in Southeast Asians. Science. 2009;326:1546-9.

24. Carter N, Pamba A, Duparc S, Waitumbi JN. Frequency of glucose-6-phosphate dehydrogenase deficiency in malaria patients from six African countries enrolled in two randomized anti-malarial clinical trials. Malar J. 2011;10:241.

25. Howes RE, Dewi M, Piel FB, Monteiro WM, Battle KE, Messina JP, et al. Spatial distribution of G6PD deficiency variants across malaria-endemic regions. Malar J. 2013;12:428.

26. Monteiro WM, Moura-Neto JP, Recht J, Bassat Q, Lacerda M. Fatal primaquine-induced hemolysis in a patient with Plasmodium vivax malaria and G6PD A(-) variant in the Brazilian Amazon. Clin Infect Dis. 2016;62:1188.

27. Deribew A, Dejene T, Kebede B, Tessema GA, Melaku YA, Misganaw A, et al. Incidence, prevalence and mortality rates of malaria in Ethiopia from 1990 to 2015: analysis of the global burden of diseases 2015. Malar J. 2017;16:271.

28. WHO. World malaria report 2012. World Health Organization, Geneva. 2013. http://www.who.int/malaria/publications/world_malaria_repor t_2012/wmr2012_full_report.pdf. Accessed 14 June 2018.

29. MoH. National strategic plan for malaria prevention, control and elimination in Ethiopia: 2014-2020. Ministry of Health, Addis Ababa. 2014. http:// www.moh.gov.et/documents/20181/21665/National+Malaria+Progr
am+Plan+2014-2020.pdf/57aaa4ca-05bf-4915-bc42-d84e0fa47a1f. Accessed 14 June 2018.

30. $\mathrm{WHO}$. Single dose primaquine as a gametocytocide in Plasmodium falciparum malaria; updated WHO policy recommendation. World Health Organization, Geneva. 2012. http://www.who.int/malaria/publications/ atoz/who_pq_policy_recommendation/en/. Accessed 14 June 2018.

31. Eziefula AC, Gosling R, Hwang J, Hsiang MS, Bousema T, von Seidlein L, et al. Rationale for short course primaquine in Africa to interrupt malaria transmission. Malar J. 2012;11:360

32. Tadesse FG, Hoogen L, Lanke K, Schildkraut J, Tetteh K, Aseffa A, et al. The shape of the iceberg: quantification of submicroscopic Plasmodium falciparum and Plasmodium vivax parasitaemia and gametocytaemia in five low endemic settings in Ethiopia. Malar J. 2017:16:99.

33. Brooker S, Kolaczinski JH, Gitonga CW, Noor AM, Snow RW. The use of schools for malaria surveillance and programme evaluation in Africa. Malar J. 2009:8:231.

34. Tadesse FG, Pett H, Baidjoe A, Lanke K, Grignard L, Sutherland C, et al. Submicroscopic carriage of Plasmodium falciparum and Plasmodium vivax in a low endemic area in Ethiopia where no parasitaemia was detected by microscopy or rapid diagnostic test. Malar J. 2015;14:303.

35. Clarke GM, Rockett K, Kivinen K, Hubbart C, Jeffreys AE, Rowlands K, et al. Characterisation of the opposing effects of G6PD deficiency on cerebral malaria and severe malarial anaemia. eLife. 2017;6:e15085.

36. Dewasurendra RL, Rockett KA, Fernando SD, Carter R, Kwiatkowski DP, Karunaweera ND, et al. G6PD gene variants and its association with malaria in a Sri Lankan population. Malar J. 2015;14:93.

37. Dombrowski JG, Souza RM, Curry J, Hinton L, Silva NRM, Grignard L, et al. G6PD deficiency alleles in a malaria-endemic region in the Western Brazilian Amazon. Malar J. 2017:16:253.

38. Harris PA, Taylor R, Thielke R, Payne J, Gonzalez N, Conde JG. Research electronic data capture (REDCap)-a metadata-driven methodology and workflow process for providing translational research informatics support. J Biomed Inform. 2009;42:377-81.

39. Barrett JC, Fry B, Maller J, Daly MJ. Haploview: analysis and visualization of LD and haplotype maps. Bioinformatics. 2005:21:263-5.

40. Manjurano A, Sepulveda N, Nadjm B, Mtove G, Wangai H, Maxwell C, et al. African glucose-6-phosphate dehydrogenase alleles associated with protection from severe malaria in heterozygous females in Tanzania. PLoS Genet. 2015;11:e1004960.

41. Al-Sweedan SA, Awwad N. Molecular characterization of glucose-6-phosphate dehydrogenase deficiency among Jordanians. Acta Haematol. 2012:128:195-202.

42. Sepúlveda N, Manjurano A, Campino SG, Lemnge M, Lusingu J, Olomi R, et al. Malaria host candidate genes validated by association with current, recent, and historical measures of transmission intensity. J Infect Dis. 2017:216:45-54.

43. Tsegaye A, Golassa L, Mamo H, Erko B. Glucose-6-phosphate dehydrogenase deficiency among malaria suspects attending Gambella hospital, southwest Ethiopia. Malar J. 2014;13:438.

44. Mourant AE, Kopeć AC, Ikin EW, Lehmann H, Bowen-Simpkins P, Fergusson ILC, et al. The blood groups and haemoglobins of the Kunama and Baria of Eritrea, Ethiopia. Ann Hum Biol. 1974;1:383-92.

45. MoH. Ethiopia National Malaria Indicator Survey 2015. Ministry of Health, Addis Ababa. 2016. https://www.ephi.gov.et/images/pictures/downl oad2009/MIS-2015-Final-Report-December-_2016.pdf. Accessed 29 Aug 2017

46. Woube M. Geographical distribution and dramatic increases in incidences of malaria: consequences of the resettlement scheme in Gambela, SW Ethiopia. Indian J Malariol. 1997;34:140-63.

47. Krafsur ES, Armstrong JC. An integrated view of entomological and parasitological observations on falciparum malaria in Gambela, Western Ethiopian Lowlands. Trans R Soc Trop Med Hyg. 1978;72:348-56.

48. Krafsur ES. Malaria transmission in Gambela, Illubabor Province. Ethiop Med J. 1971:9:75-94.

49. Wakuma Abaya S, Mandere N, Ewald G. Floods and health in Gambella region, Ethiopia: a qualitative assessment of the strengths and weaknesses of coping mechanisms. Glob Health Action. 2009;2:1.

50. Allison AC. Glucose-6-phosphate dehydrogenase deficiency in red blood cells of East Africans. Nature. 1960;186:531-2.

51. Beutler E. Glucose-6-phosphate dehydrogenase deficiency. N Engl J Med. 1991;324:169-74 
52. Carter TE, Mekonnen SK, Lopez K, Bonnell V, Damodaran L, Aseffa A, et al. Glucose-6-phosphate dehydrogenase deficiency genetic variants in malaria patients in Southwestern Ethiopia. Am J Trop Med Hyg. 2018;98:83-7.

53. Iancovici-Kidon M, Sthoeger D, Abrahamov A, Wolach B, Beutler E, Gelbart T, et al. A new exon 9 glucose-6-phosphate dehydrogenase mutation (G6PD "Rehovot") in a Jewish Ethiopian family with variable phenotypes. Blood Cells Mol Dis. 2000;26:567-71.

54. Valencia SH, Ocampo ID, Arce-Plata MI, Recht J, Arévalo-Herrera M. Glucose-6-phosphate dehydrogenase deficiency prevalence and genetic variants in malaria endemic areas of Colombia. Malar J. 2016;15:291

55. Ghimire P, Singh N, Ortega L, Rijal KR, Adhikari B, Thakur GD, et al. Glucose-6-phosphate dehydrogenase deficiency in people living in malaria endemic districts of Nepal. Malar J. 2017;16:214.

56. Chu CS, Bancone G, Nosten F, White NJ, Luzzatto L. Primaquine-induced haemolysis in females heterozygous for G6PD deficiency. Malar J. 2018;17:101.

57. Beutler E, Yeh M, Fairbanks VF. The normal human female as a mosaic of X-chromosome activity: studies using the gene for C-6-PD-deficiency as a marker. Proc Natl Acad Sci USA. 1962;48:9-16.

58. Baird JK, Dewi M, Subekti D, Elyazar I, Satyagraha AW. Noninferiority of glucose-6-phosphate dehydrogenase deficiency diagnosis by a pointof-care rapid test vs the laboratory fluorescent spot test demonstrated by copper inhibition in normal human red blood cells. Transl Res. 2015; 165:677-88.

59. Roca-Feltrer A, Khim N, Kim S, Chy S, Canier L, Kerleguer A, et al. Field trial evaluation of the performances of point-of-care tests for screening G6PD deficiency in Cambodia. PLoS ONE. 2014;9:e116143.

60. Weppelmann TA, von Fricken ME, Wilfong TD, Aguenza E, Philippe TT, Okech BA. Field Trial of the Carestart Biosensor analyzer for the determination of glucose-6-phosphate dehydrogenase activity in Haiti. Am J Trop Med Hyg. 2017;97:1262-70.

61. Ley B, Bancone G, von Seidlein L, Thriemer K, Richards JS, Domingo GJ, et al. Methods for the field evaluation of quantitative G6PD diagnostics: a review. Malar J. 2017; 16:361.

62. Thriemer K, Ley B, Bobogare A, Dysoley L, Alam MS, Pasaribu AP, et al. Challenges for achieving safe and effective radical cure of Plasmodium vivax: a round table discussion of the APMEN Vivax Working Group. Malar J. 2017:16:141

63. Getahun B, Nkosi ZZ. Is directly observed tuberculosis treatment strategy patient-centered? A mixed method study in Addis Ababa, Ethiopia. PLoS ONE. 2017;12:e0181205

64. Tadesse FG, Slater HC, Chali W, Teelen K, Lanke K, Belachew M, et al. The relative contribution of symptomatic and asymptomatic Plasmodium vivax and Plasmodium falciparum infections to the infectious reservoir in a low-endemic setting in Ethiopia. Clin Infect Dis. 2018;66:1883-91.
Ready to submit your research? Choose BMC and benefit from:

- fast, convenient online submission

- thorough peer review by experienced researchers in your field

- rapid publication on acceptance

- support for research data, including large and complex data types

- gold Open Access which fosters wider collaboration and increased citations

- maximum visibility for your research: over 100M website views per year

At BMC, research is always in progress.

Learn more biomedcentral.com/submissions 